\title{
Applying Mathematical Morphology for the Classification of Iberian Ceramics from the Upper Valley of Guadalquivir River
}

\author{
M. Lucena ${ }^{1}$, A.L. Martínez-Carrillo ${ }^{2}$, J.M. Fuertes ${ }^{1}$, F. Carrascosa ${ }^{1}$, and A. Ruiz ${ }^{2}$ \\ 1 Department of Computer Science, University of Jaen, Spain \\ mlucena@ujaen.es \\ 2 Research University Institute for Iberian Archaeology, University of Jaen, Spain \\ caai@ujaen.es
}

\begin{abstract}
Although the potential of morphometrics for the study of archaeological artefacts is recognized, quantitative evaluation of the concordance between such methods and traditional typology and the potential of these techniques as supported methodologies in the archaeological analysis is a pending task. We present a new method to characterize and classify wheel-made pottery by its profile, using Mathematical Morphology. Each piece is represented as a vector, obtained by sampling the so called morphological curves (erosion, dilation, opening and closing), and Euclidean Distance is used as a similarity measure. The proposed technique has been studied using a sample of 1133 complete ceramic vessels from the Iberian archaeological sites from the upper valley of Guadalquivir River (Andalusia, Spain), showing that it is compatible with the existing corpus, established by experts.
\end{abstract}

Keywords: Ceramic Profiles, Typologies, Shape Matching, Mathematical Morphology.

\section{Introduction and Related Work}

The study and analysis of archaeological ceramics constitutes one of the most frequent activities of the archaeological work, which consists habitually of classifying the thousands of ceramic fragments gathered in the interventions and selecting those that contribute to deduce forms, functions and chronology [1].

The different criteria used in the elaboration of classifications do not contribute to homogenize the analysis of the pottery shapes, since the election of criteria depends on each researcher and moment [2]. In this sense, Shepard saw three phases in the election of criteria: the study of whole vessels as culture-objects; the study of sherds as dating evidence for stratigraphic sequences; the study of pottery technology as a way of relating more closely to the potter, but she did not try to put dates to them.

Chronologically, the most used criteria have been artistically, typological, functional, technological, statistical, and contextual. Finally we can see developing interest in integrating ceramics into a wide analysis of finds assemblages. This must be the next step in ceramic studies: having integrated the various aspects of ceramics studies in the contextual phase (1960 and after), we must now begin to integrate ceramic studies into the wider field of general finds assemblages. 
Morphology-based classification systems (do not confuse with Mathematical Morphology, the theory in which our method is based) emphasize the attributes of the shape of a vessel. Starting from the early works of Shennan and Wilcock [3], many systems have been developed to classify vessels from the shape of its profile [4]. Some methods try to find parametric representations of the shapes [5], mainly for archiving purposes, and measurement of different volumetric features, such as height, width, diameter or volume [6]. Other methods are designed to allow the automatic comparison between profiles. In [7], a measure based on the overlap maximization between two profiles is defined, although the authors recognize that it is not well suited for vessels because of the elongated shapes of the profiles. Other works rely on local features, such as context descriptors [8], to characterize profile shapes [9], along with a multivariate analysis to compare and group profiles into clusters of similar vessels. Other authors [10, 11] propose a profile representation based on a continuous, sub-pixel approximation of the profile shape, and calculate its radius, tangent and curvature along the contour. Similarity is then computed using a weighted Euclidean Distance.

This paper presents a novel method for encoding morphological data of wheel-made pottery profiles, in order to be later recovered and compared, providing us with a tool to help the archaeologist to find in a profile database the most similar classes to a given shape. Each profile, defined as the cross section of the vessel in the direction of the rotational axis of symmetry (see Figure 1), is characterized by means of several morphological curves [12, 13] extracted from its shape, a method that has been used successfully in other shape recognition related problems [14, 15].

The Iberian pottery (S.VI B.C. - I A.C.) from the upper valley of Guadalquivir River (Spain) has been chosen for this purpose, because is a well documented region with many excavated sites [16-18]. The selected ceramic material for the morphological analysis comes from different archaeological settlements located in the provinces from Jaén, Granada and Córdoba. Most of the ceramic vessels have been documented in the province of Jaén, since they pertain to Iberian period.

This paper is organized as follows: Section 2 is devoted to explain the corpus of pottery, provided by experts, on which our experiments are based. Section 3 explains the fundamentals on morphological analysis, and how it is used in our method. Section 4 shows in detail how the profiles are characterized and classified. Experimental results and conclusions are shown in Sections 5 and 6 .

\section{The Corpus of Ceramics and the Traditional Classification}

The selected ceramic material comes from different archaeological settlements located in the East area of Andalusia, specifically the provinces from Jaén, Granada and Cordoba. Most of the ceramic vessels have been documented in the province of Jaén, since them pertaining to Iberian period (S.VI B.C. - I A.C.). In this area has been one expanded tradition with respect to the study of ceramic typologies of the Iberian period, emphasizing the works of Pereira [19] for the Iberian painted ceramics of the valley of Guadalquivir.

The combination of different archaeological sites, with different chronologies makes the accomplishment of a diachronic and synchronous study possible, allowing to contrast materials of different archaeological sites with different chronologies. 
In total, we have a database of 1133 vessel profiles, corresponding to Iberian pottery, found in 16 archaeological sites belonging to the upper valley of Guadalquivir River (Spain).

Our classification is based on morphometric criteria supervised by an expert. Several formal parts of a ceramic vessel have been differentiated (lip, neck, body, base and handles) to facilitate both the comparison between vessels. Classes are defined taking into account the presence or absence of parts, and the ratio between their sizes.

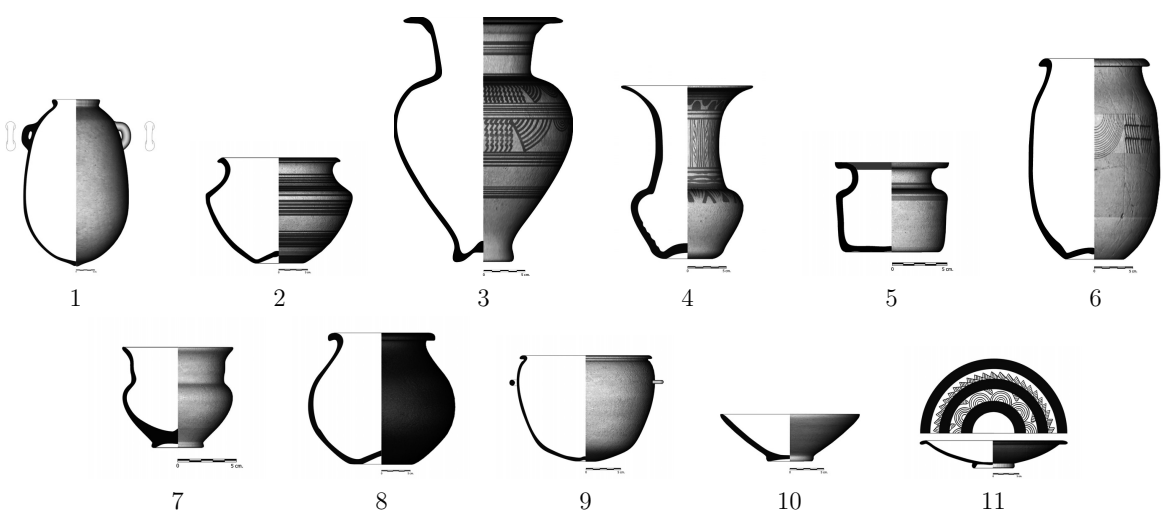

Fig. 1. Example profiles of each of the eleven classes

Following this classification criterion we have defined 11 classes (representative profiles are shown in Figure 1). The first nine correspond to closed forms, while the latter two correspond to open forms:

1. Shapes in which the body predominates over the other parts of the vessel. The shape of the body is oval.

2. Shapes in which the body has form of conic bifrustum. The joints of the different body parts are discontinuous.

3. Shapes with developed, parallel walls neck, and body globular body.

4. Shapes with developed, divergent walls neck, and globular body. This shape is also named chardon vessel.

5. Shapes with cylinder body. Also known as kalathos.

6. Lengthened shapes.

7. Shapes with profile in form of $S$.

8. Shapes with globular body. The joints of the different body parts are continuous.

9. Shapes with globular body and a largely developed rim.

10. Shapes with semi-spherical body.

11. Shapes with semi-spherical body and reversed rim. 


\section{Morphological Filtering}

Mathematical Morphology [12] is based in set theory, and provides us with a powerful approach to numerous image processing problems [13]. A morphological description of a binary image is the set of $2 \mathrm{D}$ vectors representing the coordinates of all the foreground (black in our case) pixels. One shape within an image can be represented as a random set $A$, where the probability that a point $x$ belongs to the $A$ is $p=P(x \in A)$.

Given a set $W$ of points representing the entire image, the above probability can be approximated by [20]:

$$
\bar{p}=\frac{\operatorname{area}(A \cap W)}{\operatorname{area}(W)}
$$

We define $e_{T}, d_{T}, o_{T}$ and $c_{T}$ as the erosion, dilation, opening and closing operations applied respectively to the point set $A$ by structuring element $T$. It can be clearly stated that $e_{T} \leq o_{T} \leq c_{T} \leq d_{T}$. Varying the size of $T$, we can obtain four curves from a given shape $A$ (Figure 2). These curves are monotonic with respect to the size of $T$, and represent the surface ratio variation between the area covered by the initial shape and the result of each morphological operation with different sizes of $T$.
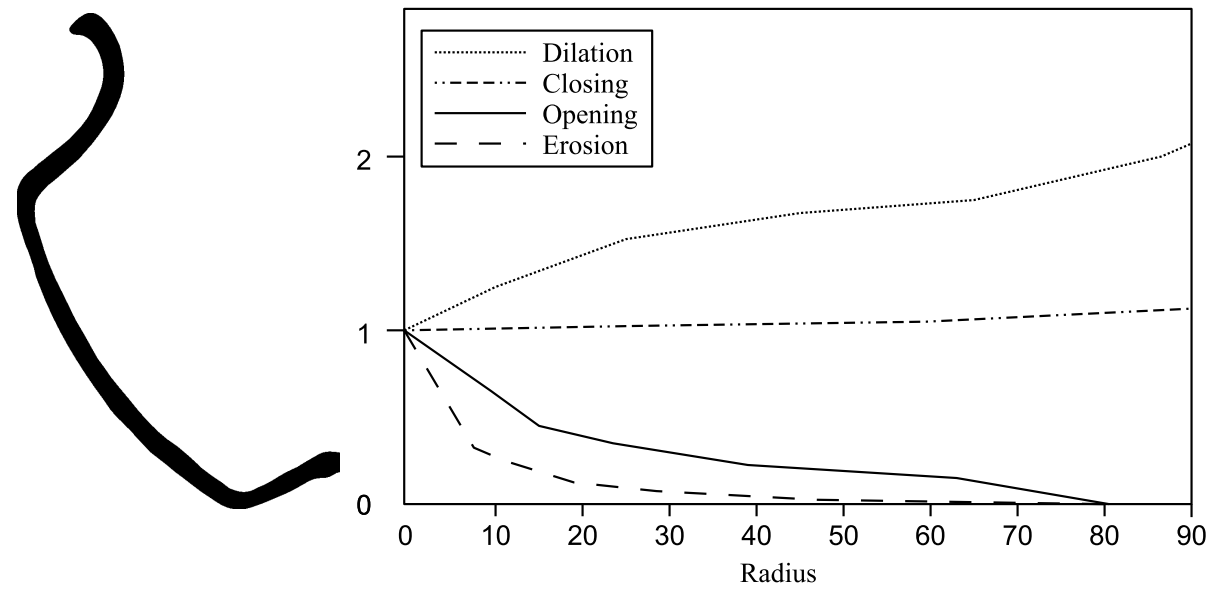

Fig. 2. Erosion, Dilation, Opening and Closing characteristic curves of a profile of the database, using an isotropic (circular) structuring element, normalized by the area of the profile

We will use the curves we have just defined, computed with different structuring elements, to obtain a characteristic vector for each profile in our database, starting from a binary image representing the shape of the corresponding vessel. We will describe in detail the full process in the following section. 


\section{Profile Characterization}

To obtain a characteristic vector for each profile, we will follow a three stage process: a pre-processing step, where we split the profile into several parts; a morphological step, where the four characteristic curves are computed with several structuring elements for each part; and finally the characteristic vector computation itself.

\subsection{Pre-processing}

In order to be processed, we start by splitting each profile it into several parts and compute the characteristic curves from each of them. We will define $d$ as the length of the line segment joining the upper point of the profile rim $\mathbf{u}$ with the lowest point of the base $\mathbf{v}$ (Figure 3). We also denote $\mathbf{p}$ as the lowest profile point located at the rotation axis. Three sub-profiles are then defined:

- Rim: Points whose distance to $\mathbf{u}$ is lower than $d / 3$.

- Base: Points whose distance to $\mathbf{p}$ is lower than the distance between $\mathbf{v}$ and $\mathbf{p}$.

- Body: The rest of the points.

If the horizontal distance between $\mathbf{v}$ and $\mathbf{p}$ is lower than their vertical distance, we consider that the base sub-profile is empty.

Our base and body parts correspond respectively to the base and body parts defined by the experts, while our rim part corresponds approximately to the union of lip, neck, and eventually the handle, if present. In the experimental section, we show results with characteristic curves for rim, and body parts, as these are the ones that are used by experts to assign a class to a given profile.

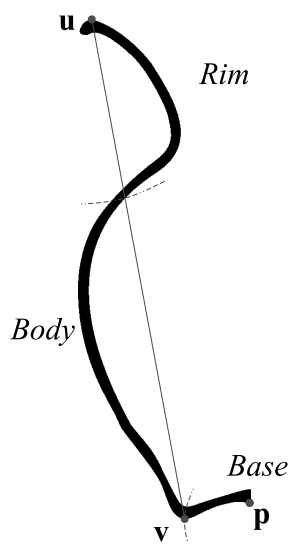

Fig. 3. Segmentation of a profile into rim, body and base parts 


\subsection{Morphological Curves}

In order to capture features with different orientation, we will use several anisotropic structuring elements. Specifically, a centred, variable length line with a 1-pixel constant width, with two parameters: an orientation angle, and the length of the line itself.

To align the points where the characteristic curves will be sampled, we have used the following strategy: first, we obtain the minimal length $l$ for the structuring element that completely eliminates the shape by erosion, and then sample the erosion, dilation, opening and closing curves with a set of lengths obtained by multiplying $l$ by several fixed coefficients. This procedure makes our technique scale invariant. Finally, once computed, each curve is normalized by the area of the initial profile so that it gives us a value of 1 .

\subsection{Characteristic Vector}

After segmenting the initial profile into sub-profiles, the four morphological curves are computed and sampled from each sub-profile and anisotropic structuring element, giving us a series of coefficients that we assemble into a single vector. With two subprofiles, $N$ angles for the structuring element, and four curves sampled in $Q$ points we obtain for each profile a vector of $2 \times N \times 4 \times Q$ components.

In our experiments, we have used $N=16$ different orientations for the structuring element. Also, the set of coefficients that multiply the minimal length $l$ previously defined is $\{0.15,0.2,0.3,05,0.75,1\}$, so $Q=6$. Therefore, we have a vector of length 768 for each profile.

\section{Experiments}

Our database has 1133 profiles, grouped into 11 classes, ranging from 10 to 373 elements each. Profiles are represented as white background PNG binary images, ranging from 0.5 to 2 megapixels in size, proportional to the actual size of the corresponding vessel. However, the size of the images is not relevant, as long as they have enough resolution, as the lengths of the structuring elements are scaled independently for each one.

In all of our experiments, similarity between two profiles has been computed as the Euclidean Distance between their associated vectors. Firstly, we will show the first 10 nearest neighbours of some sample profiles, along with the measured similarity for each one (Figures 4 to 6). As can be seen, the recovered profiles correspond with the typologies defined by the experts.

We have also carried out a classification experiment, using a leave-one-out cross validation strategy. For a given profile, we select the class that appears more times among the $M$ nearest neighbours. Results with one, three and five neighbours are shown in Table 1. We have compared our results with the ones obtained by the method proposed in [11], obtaining clearly better recognition rates. We can also see that increasing the number of neighbours does not improve classification rates. 

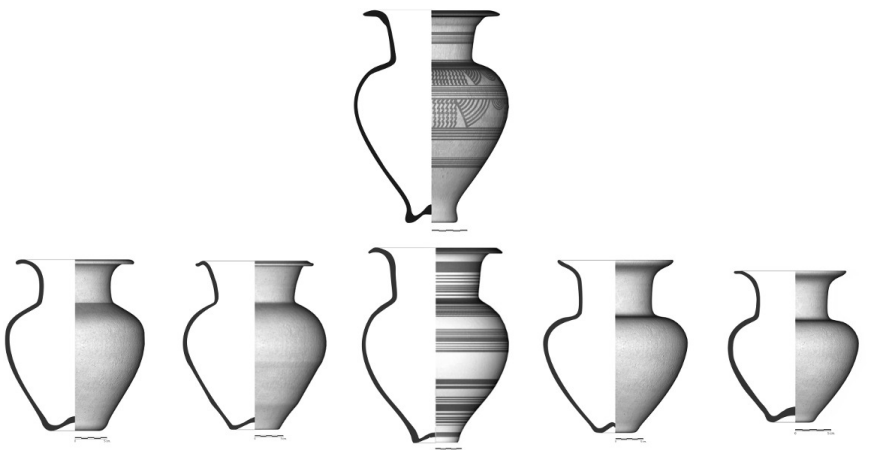

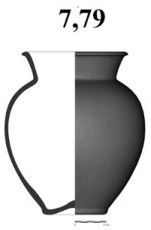

11,38

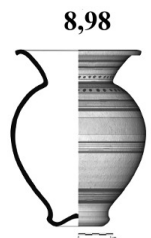

11,61

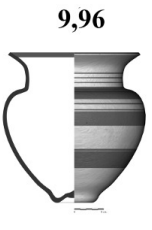

11,64

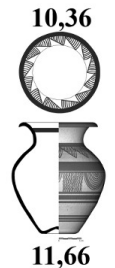

10,59

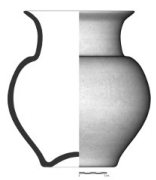

11,77

Fig. 4. Most similar shapes to a given example, using our Mathematical Morphology based similarity distance. Similarity distances are shown under each piece.

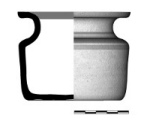

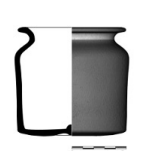

8,35

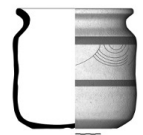

10,07

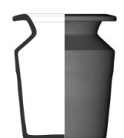

8,85

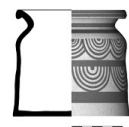

10,14

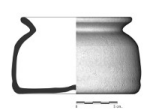

9,49

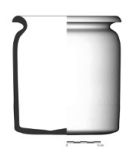

10,22

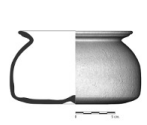

9,85

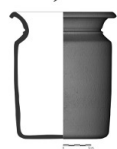

10,32

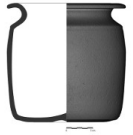

9,97

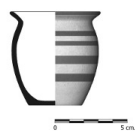

10,41

Fig. 5. Most similar shapes to a given example, using our Mathematical Morphology based similarity distance. Similarity distances are shown under each piece.

Table 1. Classification rates obtained by our method and the one proposed by Karasik et al. [11] and previously propounded by Saragusti et al. [10], with 1, 3 and 5 neighbours.

\begin{tabular}{|c|c|c|}
\hline Neighbours (M) & Morphological Vector & Karasik et al. [11] \\
\hline \hline $\mathbf{1}$ & $\mathbf{8 5 . 7 0 \%}$ & $76.88 \%$ \\
\hline $\mathbf{3}$ & $84.20 \%$ & $74.93 \%$ \\
\hline $\mathbf{5}$ & $84.47 \%$ & $74.23 \%$ \\
\hline
\end{tabular}




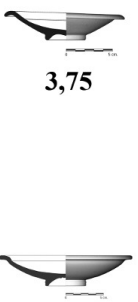

3,98
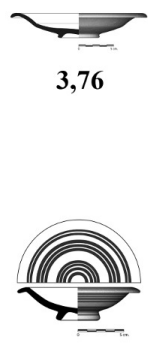

4,27

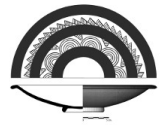

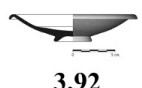

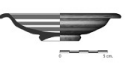

4,27
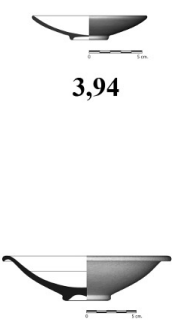

4,33
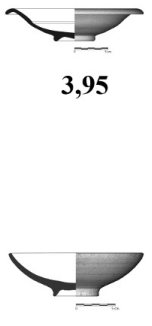

4,36

Fig. 6. Most similar shapes to a given example, using our Mathematical Morphology based similarity distance. Similarity distances are shown under each piece.

Figure 7 show the normalized confusion matrix with one neighbour. Each row contains all the profiles of a single class, while each column contains all the profiles that our method assigns to the corresponding class.

We can note that our method classifies wrongly many class 2 (biconical trunk) and class 9 (globular with large rim) profiles as class 8 (globular), but not conversely. This is possibly due to the small size of classes 2 ( 47 samples) and 9 ( 22 samples) versus class 8 (293 samples), together with the fact that these three classes are morphologically very similar.

\begin{tabular}{r|cccccccccccc|}
\multicolumn{10}{c|}{ Rim - Body } \\
1 & 83.33 & 0 & 0 & 0 & 0 & 3.33 & 0 & 13.33 & 0 & 0 & 0 \\
2 & 0 & 42.55 & 0 & 0 & 0 & 0 & 4.26 & 53.19 & 0 & 0 & 0 \\
3 & 0 & 2.67 & 72.00 & 4.00 & 0 & 1.33 & 2.67 & 16.00 & 0 & 0 & 0 \\
4 & 0 & 0 & 0 & 80.00 & 0 & 0 & 10.00 & 10.00 & 0 & 0 & 0 \\
5 & 0 & 0 & 0 & 0 & 100.00 & 0 & 0 & 0 & 0 & 0 & 0 \\
6 & 0 & 0 & 1.92 & 0 & 3.85 & 78.85 & 0 & 13.46 & 1.92 & 0 & 0 \\
7 & 0 & 0 & 0 & 0 & 0 & 2.00 & 86.00 & 4.00 & 2.00 & 6.00 & 0 \\
8 & 1.37 & 3.41 & 2.39 & 0 & 0 & 1.37 & 0.34 & 89.42 & 1.37 & 0.35 & 0 \\
9 & 0 & 0 & 0 & 0 & 0 & 9.09 & 0 & 40.91 & 45.45 & 4.55 & 0 \\
10 & 0 & 0.54 & 0.27 & 0 & 0 & 0 & 0 & 0 & 0.27 & 94.37 & 4.29 \\
11 & 0 & 0 & 0 & 0 & 0 & 0 & 0 & 0 & 0.80 & 19.20 & 80.00 \\
\hline
\end{tabular}

Fig. 7. Normalized confusion matrix resulting of the application of our method to the database, using the rim and body sub-profiles 


\section{Conclusions}

The above-mentioned investigations demonstrate that in the field of archaeological investigation of pottery the collaboration between archaeologist and computer scientists permits the development of useful applications for the classification of archaeological ceramics.

We have proposed a new method to classify vessel profiles, based on well known mathematical operators, that captures most of their morphometric features, allowing us to identify the most similar profiles in a database. Through this methodology for measuring the similarity between ceramic profiles it is possible the search of parallels in a particular region, that in this case is the upper valley of Guadalquivir River (Spain), showing the concordance between morphometrics and traditional typology.

Our vector characterization captures the morphological features of the profiles. The results are clearly better than the ones obtained by other, state of the art methods. Although, unlike others, our representation does not allow to reconstruct the profile shape, the graphical data that we use as input is compact enough to be stored together with the vector itself.

As a future work, it would be interesting to include the base part of the profiles into the characteristic vector, and compare the results to know if it gives some improvement. Other interesting work would be the use of our method to obtain an automatic clustering of the database, and compare the resulting classes to the ones defined by the experts. The proposed technique can also be used with other similar profile databases, in order to better test its applicability.

Acknowledgement. This work has been supported by the Excellent Projects Program of CICE (regional government), the European Union ERDF funds under research projects P07-TIC-02773, the Computer Graphics and Geomatics Research Group (TIC144) of the University of Jaén, and the Andalusian Economics, Innovation, Science and Employment Council under project TIC-7278.

\section{References}

1. Orton, C., Tyers, P., Vince, A.: Pottery in Archaeology. Cambridge University Press (1993)

2. Shepard, A.: Ceramics for the archaeologist. Carnegie Institution of Washington (1956)

3. Shennan, S., Wilcock, J.: Shape and style variation in central german bell beakers. Science and Archaeology 15, 17-31 (1975)

4. Rice, P.M.: Pottery Analysis. University of Chicago Press, Chicago (1987)

5. Nautiyal, V., Kaushik, V.D., Pathak, V.K., Dhande, S., Nautiyal, S., Naithani, M., Juyal, S., Gupta, R.K., Vasisth, A.K., Verna, K.K., Singh, A.: Geometric modeling of indian archaeological pottery: A preliminary study. In: Clark, J., Hagemeister, E. (eds.) Exploring New Frontiers in Human Heritage, CAA 2006, Fargo, United States. Computer Applications and Quantitative Methods in Archaeology (2006)

6. Kampel, M., Sablatnig, R.: An automated pottery archival and reconstruction system. Journal of Visualization and Computer Animation 14(3), 111-120 (2003)

7. Mom, V.: Where did i see you before. holistic method to compare and find archaeological artifacts. In: Decker, R., Lenz, H. (eds.) Advances in Data Analysis. Proceedings of the 30th Annual Conference of the Gesellschaft fr Klassifikation. Springer, Berlin (2006) 
8. Belongie, S., Malik, J., Puzicha, J.: Shape matching and object recognition using shape contexts. IEEE Transactions on Pattern Analysis and Machine Intelligence 24(4), 509-522 (2002)

9. Maaten, L., Lange, G., Boon, P.: Visualization and automatic typology construction of pottery profiles. In: Frischer, B. (ed.) Making history interactive: computer applications and quantitative methods in archaeology (CAA). BAR International Series, vol. 2079, Archaeopress, Oxford (2009)

10. Saragusti, I., Karasik, A., Sharon, I., Smilansky, U.: Quantitative analysis of shape attributes based on contours and section profiles in artifact analysis. Journal of Archaeological Science 32(6), 841-853 (2005)

11. Karasik, A., Smilansky, U.: Computerized morphological classification of ceramics. Journal of Archaeological Science 38(10), 2644-2657 (2011)

12. Matheron, G.: Random Sets and Integral Geometry. Wiley (1975)

13. Haralick, R., Sternberg, S., Zhuang, X.: Image analysis using mathematical morphology. IEEE Transactions on Pattern Analysis and Machine Intelligence 9(4), 532-550 (1987)

14. Fuertes, J., Lucena, M., Pérez de la Blanca, N., Fdez-Valdivia, J.: Combining morphological filters and deformable models to desing a $2 \mathrm{~d}$ shape based retrieval system. In: 12th Scandinavian Conference on Image Analysis (SCIA 2001), Bergen (Norway), vol. 1, pp. 646-653 (June 2001)

15. Fuertes, J., Lucena, M., Pérez de la Blanca, N., Ruiz, N.: Objects matching combining color and shape. In: 4th EURASIP Conference focused on Video/Image Processing and Multimedia Communications, vol. 1, pp. 201-208 (2003)

16. Chapa, T., Pereira, J., Madrigal, A., Mayoral, V.: La Necrópolis ibérica de Castellones de Ceal (Hinojares, Jaén). Consejería de Cultura. Junta de Andalucía (1997)

17. Ruiz Rodríguez, A., Molinos, M., López, J., Crespo, J., Choclán, C., Hornos, F.: El horizonte ibérico antiguo del Cerro de la Coronilla (Cazalilla, Jaén). Cortes A y F. Cuadernos de Prehistoria de la Universidad de Granada (8), 251-295 (1983)

18. Ruiz Rodríguez, A., Hornos Mata, F., Choclán, C., Cruz Garrido, J.: La necrópolis ibérica Finca Gil de Olid (Puente del Obispo-Baeza). Cuadernos de Prehistoria de la Universidad de Granada (9), 195-234 (1984)

19. Pereira Sieso, J.: La cerámica ibérica de la cuenca del Guadalquivir. Trabajos de Prehistoria 46, 149-159 (1989)

20. Serra, J.: Image Analysis and Mathematical Morphology. Academic Press, Inc., Orlando (1983) 